\title{
New or Noteworthy Plants from China I
}

\section{Hisao Migo*}

Received March-11, 1942.

1. Oenanthe javanica DC. var. elongata (Metcalf) Migo, comb. nov.

Oenanthe linealis Wall. Cat. no. 586 (1828-32); DC. Prodr. IV, p. 136 (1830); Matsumura \& Hayata, Enum. Pl. Formosa. p. 172 (1906); Chermezon in Lecomte, Fl. Gén. Indo-Chine, II, p. 1147 (1923) ; HandelMazzetti, Symb. Sin. VII, p. 722 (1933).

Oenanthe sinensis DunN in Journ. Linn. Soc. XXXV, p. 496 (1903); HaNDel-Mazzetti, Symb. Sin. VII, p. 722 (1933).

Oenanthe stolonifera form. elongata Metcalf in Lingnan Sci. Journ. XIII, p. 518 (1938).

Hab. Formosa: Tomita-chô, Taihoku-shi (T. Tanaka \& Y. Shimada, Apr. 15, 1933).

China :

Kiangsu-Chinkiang (H. Mıю, Jun. 30, 1934); pede montis Shangfang-shan, Soochow (H. Migo, Jul. 2, 1933) ; Kwanyin-shan, Soochow (H. Migo, Jun. 3, 1933).

Anhwei-Anking (H. Migo, Apr. 18, 1941).

Kiangsi-Kiukiang (H. Migo, Apr. 29, 1941).

Distr. India, Indochina, China \& Formosa.

Most authors in dealing with this variety and its type species have attached importance to the shape of the leaves, but it appears to me very doubtful if this character has any real significance for specific separation, since the leaves sometimes vary considerably from ovate to linear even on the same individual. The type specimen of $O$. sinensis, of which $\mathrm{I}$ have seen a photograph, is an extreme form with the linear leaves.

2. Peucedanum japonicum Thunberg, Fl. Jap. p.117 (1784) ; MasaMUNE, Fl. Geobot. Stud. Yakusima, p. 339 (1934).

Hab. Chekiang-Insl. Puto (H. Migo, Jun. 5, 1936).

Distr. Korea, Japonia, Formosa \& China.

This is new to the flora of China.

3. Omphalodes chekiangensis Mrgo, sp. nov.

* The Shanghai Science Institute, Shanghai, China. 
Rhizoma breve crassum usque ad $5 \mathrm{~cm}$ longum $0.6 \mathrm{~cm}$ in diametro, apice cum reliquis petiolorum et caulorum emortuorum instructum, et radices numerosos fibrillosos emittens. Caules caespitosi paucifoliati debiles arcuato-adscendentes $10-20 \mathrm{~cm}$ alti hirsuti, post anthesin usque ad $40 \mathrm{~cm}$ elongati et apice radicantes. Folia radicalia longe petiolata ubique hirsuta ; petiolis sub anthesin 5-10 cm longis sub fructu usque ad $20 \mathrm{~cm}$ elongatis basi valde dilatatis; laminis papyraceis ovatis $6 \times 3 \mathrm{~cm}-12 \times 7 \mathrm{~cm}$ apice breviter acuminatis margine obsolete denticulatis basi cordatis vel cordatotruncatis praesertim sub fructu profunde cordatis; folia caulina radicalia simulantia sed minora et petiolis brevioribus. Cincinni laterales interdum terminales breves 10-20-flori densissimi sub fructu non vel paulo elongati $3-5 \mathrm{~cm}$ longi, pedicellis inferioribus calycibus longioribus sed superioribus brevioribus ebracteatis dense hirsutis. Calyx usque ad basin 5-partitus, segmentis lanceolatis $5 \times 1.5 \mathrm{~mm}$ apice acutis dorso albo-hirsutis, post anthesin usque ad $8 \times 3 \mathrm{~mm}$ accrescentibus. Corolla $8 \mathrm{~mm}$ longa glabra 5-lobata alba, tubo $5 \mathrm{~mm}$ longo $2.5 \mathrm{~mm}$ in diametro, lobis rotundato-ovatis subpatentibus $3 \mathrm{~mm}$ longis $2.5 \mathrm{~mm}$ latis. Fornices subquadrati paulo latiores quam longi emarginati papillosi. Stylus calycem subaequans sed post anthesin superans glaber filiformis $5 \mathrm{~mm}$ longus, ovariis 4 glabris. Stamina 5 exerta, filamentis glabris medio tubi affixa, antheris oblongis ca. $1 \mathrm{~mm}$ longis. Nuculae maturae pateriformes nigricantes lucidae, marginibus simplicibus integris albidis ca. $3 \mathrm{~mm}$ in diametro.

Hab. Chekiang-Mt. Hsi-tienmu-shan (H. Migo, Mai. 15, 1935-typus fr.) ; ibid. (H. Migo, Apr. 23, 1936-typus fl. in Herb. Inst. Sci. Shanghaiensis).

Distr. Planta endemica.

O. moupinense affinis sed exqua differt inftorescentibus densissimis, floribus albis, stylis calicibus aequilongis et staminibus exertis.

\section{Scutellaria glecomoides Mrgo, sp. nov.}

Caulis debilis adscendens tetragonus $10-25 \mathrm{~cm}$ altus $1-1.5 \mathrm{~mm}$ crassus araneoso-puberulus simplex. vel e basi ramosus, stolones longos tuberiferos emittens. Tuber globosum vel ovoideum $5-8 \mathrm{~mm}$ in diametro. Folia opposita longe petiolata; radicalia $3-4 \mathrm{~cm}$ longa sed sursum sensim diminuta puberula, laminis orbicularibus vel ovatis interdum reniformibus $1-2 \mathrm{~cm}$ in diametro, margine subregulariter crenatis basi profunde cordatis, crenis utrinque 4-7, supra viridibus subtus glaucescentibus interdum purpurascentibus utrinque pubescentibus palmatinerviis. Folia floralia caulinis conformia. Flores solitarii axillares breviter pedicellati. Calyx campanulatus $3 \mathrm{~mm}$ longus $2 \mathrm{~mm}$ in diametro breviter bilabiatus saepe purpurascens extus pubescens intus glaber, lobo superiore dorso scutello sublunato instructo. 
Corolla coerulea sed basi albescens anguste tubulosa 1-1.2 cm longa basi $2 \mathrm{~mm}$ apice $4-5 \mathrm{~mm}$ in diametro extus intusque paulo pubescens, labio superiore breviore paulo galeato trilobato, inferiore superiore fere duplo longiore patento-dilatato late obovato ca. $8 \mathrm{~mm}$ longo margine irregulariter repando. Stamina 4 didynama, filamentis filiformibus glabris dilute coeruleis, antheris reniformibus ciliatis atrocoeruleis. Stylus $7 \mathrm{~mm}$ longus glaber apice recurvatus purpurascens. Ovarium aequaliter 4-partitum late obovoideum compressum glabrum.

Hab. Kiangsu-Mt. Shangfang-shan prope Soochow (H. Migo, Apr. 5, 1935-typus in Herb. Inst. Sci. Shanghaiensis).

Distr. Planta endemica.

This is an interesting species resembling Glecoma hederacea L. in foliage and producing the tuber at the end of the stolon.

5. Bobua anomala (Brand) Migo, comb. nov.

Symplocos anomala BRAND in ENGLER, Bot. Jahrb. XXIX, p. 529 (1900) ; ibid. in ENGLer, Pflanzenreich, Heft 6, p. 67 (1901); ReHder in SARgent, Pl. Wilson. II, p. 596 (1916) ; ibid. in Journ. Arn. Arb. VII, p. 188 (1927) \& XV, p. 300 (1930).

Symplocos Dielsii Léveillé in Fedde, Rep. IX, p. 445 (1911); ibid. Fl. Kouy-Tchéou, p. 409 (1915).

Symplocos Esquirolii LÉveILLÉ in loc. cit. p. 445 (1911); ibid. loc. cit. p. 409 (1915).

Hab. Chekiang-Lingyin, Hangchow (H. Mrao, Mai. 23 \& Sept. 19, 1935).

Distr. China australis.

6. Bobua austrosinensis Migo, sp. nov.

Affinis B. pseudolancifolia Hatusima, sed ramulis foliisque firmioribus et glabris, calycibus glabris exqua distincta.

Arbor $4 \mathrm{~m}$ alta (e coll.). Rami et ramuli subteretes fusco-nigrescentes glabri. Gemmae glabrae. Folia breviter petiolata, petiolis 5-10 mm longis supra sulcatis glabris, laminis lanceolatis vel oblongo-laneeolatis $5-8 \mathrm{~cm}$ longis $2-3 \mathrm{~cm}$ latis basi angustatis apice caudato-acuminatis in siceo brunneo-flavescentibus subnitidis coriaceis utrinque glabris, costis supra impressis infra prominentibus, margine dentato-serrulatis. Racemi simplices axillares 2-3 cm longi 5-12-flori, rachidibus fusco-hirsutis. Bracteae scariosae dorso paulo pubescentes: bracteolae caducae. Pedicelli breves 1-1.5 mm longi hirsuti. Calyx glaber, lobis deltoideo-ovatis $1.5 \mathrm{~mm}$ longis tubum aequantibus. Corolla alba fragrans (e coll.) $3.5 \mathrm{~mm}$ longa. Stamina ca. 25. Ovarium glabrum. 
Nom. Chin. Shek Lei.

Hab. Kwangtung-near Kong Ts'uen, little North River (F. A. McClure, No. 13164, Jan. 16, 1925-typus in Herb. Inst. Sci. Shanghaiensis).

Distr. Endemica in China australi.

The specimen cited above was distributed a few years ago from Lingnan University, Canton, under the name S. caudata WALL.

7. Bobua congesta (Bentham) Migo, comb. nov.

Symplocos congesta BenthaM, Fl. Hongk. p. 211 (1861); Brand in ENGLER, Pflanzenreich, Heft 6, p. 69(1901) ; DunN \& Tutcher, Fl. Kwangt. Hongk. p. 163 (1912); Merrill in Journ. Arn. Arb. XX, p. 353 (1939).

Hab. Fukien-Mt. Ku-shan prope Foochow (H. Migo, Jul. 2, 1937).

Distr. Indochina \& China australis.

8. Bobua eriobotryaefolia (Hayata) Kanehira \& SASAKI in SASAKI, List Pl. Formosa, p. 330 (1928).

Symplocos neriifolia (non Sieb. \& Zucc.) Hemsley in Journ. Linn. Soc. XXVI, p. 73 (1889) forsan pro parte; BRAND in ENGLER, Pflanzenreich, Heft 6, p. 67 (1901) forsan pro parte ; DunN \& Tutcher, Fl. Kwangt. Hongk. p. 163 (1912) ; MerRILL in Lingnan Sci. Journ. IX, p. 43 (1930); Guillaumin in Lecomte, Fl. Gén. Indo-Chine, III, p. 1011 (1933).

Symplocos eriobotryaefolia Hayata Icon. Pl. Formosa. V, p. 98, fig. 26 \& tab. X (1915) ; Makıno \& Nemoto, Fl. Jap. ed. 2, p. 919 (1931); Mori in Sylvia, V, p. 227 (1934); Kanehira, Formosan Trees, rev. ed. p. 584, fig. 541 (1936).

Hab. Kwangtung-Tai Mo Shan, Tapu District (W. T. Tsang, No. 21015, Jun. 26-28, 1932) ; ibid. (W. T. Tsang, No. 21219, Jul. 18, 1932).

Hainan-Pak Sik Ling and vicinity, Ku Tung Village, Ching Mai District (C. I. LEI, No. 617, Mai. 4, 1933).

Distr. Formosa, China australis \& Indochina.

Judging from the description given by Guillaumin, there can be little doubt that $S$. neriifolia from Indochina of which I have seen no specimen belongs here.

9. Bobua Groffii (MerriLl) Migo, comb. nov.

Symplocos Groffii MerRILL in Philip. Journ. Sci. XII, p. 107 (1917).

Symplocos lungtauensis Merrill in Journ. Arn. Arb. VIII, p. 14 (1927).

Hab. Kwangtung-Lung Tau Shan (To \& Ts'ANG, No. 12066, Mai. 23, 1924); Chong Uen Shan near Kau Fung, Loh Ch'ang District (W. T. 
Tsang, No. 20776, Nov. 2-30, 1932).

Distr. China australis.

I am unable to find any valid differences between MerriLl's two species and it would appear that he must have overlooked his $S$. Groffii when describing S. lungtauensis.

10. Bobua stellaris (Brand) Migo, comb. nov.

Symplocos neriifolia (non Sieb. \& Zucc.) Hemsley in Journ. Linn. Soc. XXVI, p. 73 (1889) saltem pro parte; Matsuda in Bot. Mag. Tokyo, XXVI, p. 331 (1912); LiNGELsheim \& WinkLER in Limpricht, Bot. Reis. Hochgebirg. China. Ost-Tib. p. 462 (1922).

Symplocos stellaris BRAND in ENGLER, Bot. Jahrb. XXIX, p. 528 (1900) ; ibid. in ENGLer, Pflanzenreich, Heft 6, p. 68 (1901); REHDER in Sargent, Pl. Wils. II, p. 597 (1916) ; ibid. in Journ. Arn. Arb. VIII, p. 189 (1927) \& XV, p. 301 (1934).

Symplocos Wilsoni HeMssey in Kew Bull. Misc. Inform. 1906, p. 161 (1906).

Symplocos Dunniana LéveILlé in Fedde, Rep. IX, p. 445 (1911); ibid. Fl. Kouy-Tchéou, p. 409 (1915).

Litsea Chaffanjonii LEveILle in FedDe, Rep. XII, p. 182 (1913); ibid. Fl. Kouy-Tchéou, p. 220 (1914).

Hab. Chekiang-Tientung-ssu prope Ningpo (H. Migo, Jun. 21, 1936) ; Linghai (H. MIgo, Nov. 6, 1935) ; prope Kuoching-ssu, pede montis Tientai-shan (H. Mıgo, Oct. 2, 1935); Mt. Peikiao-feng, Hangchow (H. Migo, Oct. 20, 1934) ; Shang-tienchu, Hangchow (H. Migo, Apr. 17, 1935); Mt. Mokan-shan (H. Migo, Jun. 28, 1931) ; Mt. Hsi-tienmu-shan (H. Migo, Apr. 23, 1936).

Anhwei-Mt. Hwang-shan (T. J. Liv, Aug. 13, 1936).

Kiangsi-Mt. Lu-shan, inter Lienhwatung et Kuling (H. Migo, Apr. 27, 1941).

Fukien-Peiling prope Foochow (H. Migo, Apr. 10, 1937).

Kwangtung-Chou Uen Shan near Kau Fung, Loh Ch'ang District (W. T. Tsang, No. 20982, Dec. 1-28, 1932).

Distr. China australis.

11. Bobua Swinhoeana (Hance) Migo, comb. nov.

Symplocos Swinhoeana HaNce in Ann. Sei. Nat. Paris, 4 sér. XV, p. 226 (1861).

Symplocos caudata (non WALL.) Brand in ENGLER, Pflanzenreich, Heft. 6, p. 42 (1901) pro parte; Rehder in Sargent, Pl. Wils. II, p. 595 (1916) pro parte; Lingelsheim \& Winkler in Limpricht, Bot. Reis. Hoch- 
gebirg. China. Ost-Tib. p. 462 (1922).

Symplocos prunifolia (non Sieb. et Zucc.) Hemsley in Journ. Linn. Soc. XXVI, p. 74 (1889) pro parte.

Hab. Chekiang-Linghai (H. Migo, Nov. 5, 1935).

Fukien-Sütou-hsiang prope Foochow (H. Migo, Mai. 23, 1937); Peiyuan-hsiang prope Foochow (H. Migo, Jun. 25, 1937).

Kwangtung-Tai Mo Shan, Tapu District (W. T. Tsang, No. 21063, Jul. 3, 1932).

Distr. China australis.

\section{支那植物考察 I}

御江 久 夫

1. レとばぜり八獨立種トスル人ガ多イガ葉形以外ニコレトイフ特徴ガ見當ラナ イノデ變種ニ下シタ。

2. 任たんばうふう八未ダ支那二有ル事ガ制ツテ居ナカツタガ, 筆者八先年舟山群 島ノ普陀山島デ得夕。

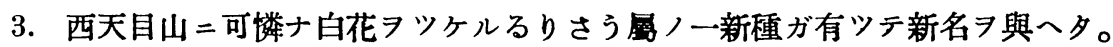

4. カナリ以前, 筆者カ蘇州/上方山デ探ツタたつなみさう感植物デ, 地下薙ノ先

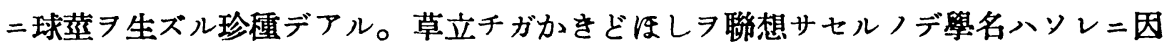
ンデ附ケタ。

5-11. 何レモ唯 Symplocos 尹 Bobua =置キ換へタダケノ事デアルガ, 文献ガ案 外錯棕シテ居ルノデ出來ルダケ整理シテ見タ。其ノ中, 6 八初島氏ガ先年 B. pseudolancifolia（南支二八極メテ普通）ト命名サレタモノニ近イ新種デアル。又, 8 八今迄 薹灣/固有種卜稱七ラレテ居タびはばみみつばひノ事デ，南支デ從來みみつばひ= 當テラレテ居タモノノ一部八本種デアル事ガ判ツタ。コレハ亦佛印 =モ有ルラシ イ。みみつばひガ南支二有ルトノ說八未ダ資料不十分デ否定八出來ナイガ，カナリ 疑ハシイ樣二思フ。假令有ツテモ稀デアラウ。 